\title{
The ultraviolet behavior of quantum gravity
}

\author{
Damiano Anselmi and Marco Piva \\ Dipartimento di Fisica "Enrico Fermi", Università di Pisa, \\ Largo B. Pontecorvo 3, 56127 Pisa, Italy \\ INFN, Sezione di Pisa, \\ Largo B. Pontecorvo 3, 56127 Pisa, Italy \\ E-mail: damiano.anselmi@unipi.it, marco.piva@df.unipi.it
}

ABSTRACT: A theory of quantum gravity has been recently proposed by means of a novel quantization prescription, which is able to turn the poles of the free propagators that are due to the higher derivatives into fakeons. The classical Lagrangian contains the cosmological term, the Hilbert term, $\sqrt{-g} R_{\mu \nu} R^{\mu \nu}$ and $\sqrt{-g} R^{2}$. In this paper, we compute the one-loop renormalization of the theory and the absorptive part of the graviton self energy. The results illustrate the mechanism that makes renormalizability compatible with unitarity. The fakeons disentangle the real part of the self energy from the imaginary part. The former obeys a renormalizable power counting, while the latter obeys the nonrenormalizable power counting of the low energy expansion and is consistent with unitarity in the limit of vanishing cosmological constant. The value of the absorptive part is related to the central charge $c$ of the matter fields coupled to gravity.

Keywords: Models of Quantum Gravity, Beyond Standard Model, Renormalization Regularization and Renormalons

ARXIV EPRINT: 1803.07777 


\section{Contents}

1 Introduction 1

2 Quantum gravity in the Batalin-Vilkovisky formalism 2

3 Renormalization 5

4 Absorptive part $\quad 8$

5 Conclusions and outlook $\quad 14$

\section{Introduction}

The problem of quantum gravity is the difficulty to reconcile renormalizability and perturbative unitarity. A solution has been recently proposed [1] by turning the ghosts due to the higher derivatives into fakeons [2], or fake degrees of freedom, which contribute to the correlation functions, but disappear from the physical spectrum. The idea amounts to a novel prescription to treat the poles of the free propagators. It is suggested by the reformulation of the Lee-Wick models [3-6] as nonanalytically Wick rotated Euclidean theories $[7,8]$. The fakeons clarify the properties of these models and refine their original, incomplete formulation. At the same time, they have a broader range of applications, to the extent that they can be introduced in models that are not of the Lee-Wick type.

Several options for quantum gravity emerge from this approach. A unique one among them is strictly renormalizable [1]. Its classical Lagrangian contains the cosmological term $\sqrt{-g}$, the Hilbert term $\sqrt{-g} R$ and the quadratic terms $\sqrt{-g} R_{\mu \nu} R^{\mu \nu}$ and $\sqrt{-g} R^{2}$. The other options are infinitely many and super-renormalizable, which makes them less attractive from the physical point of view. The proof of perturbative unitarity can be carried out to the very end [2], once the effects of the cosmological constant are neglected. The reason is that a satisfactory scattering theory has not been developed, yet, in the presence of a cosmological constant (see refs. [9, 10] for some investigations and proposals on this issue) and it might even not exist. If that were the case, a nonvanishing cosmological constant (generically turned on by the radiative corrections) would signal a unitarity anomaly in the universe, which would explain why this quantity is so small. Super-renormalizable theories of quantum gravity where the cosmological constant is not turned on by the radiative corrections can be built [1], yet it is hard to argue that they describe the laws of physics.

We think that the strictly renormalizable option is at present the best candidate to explain quantum gravity, even if the cosmological constant cannot be turned off to all orders. In this paper, we compute the key quantities of this theory at one loop. Specifically, we work out the absorptive part of the graviton self energy and the one-loop renormalization, both in the pure theory and in the presence of matter.

It can be shown $[1,2]$ that the fakeon prescription does not affect the renormalization, which coincides with the one of the Euclidean version of the theory. The one-loop beta 
functions can be calculated by working out the divergent parts of the two graviton and three graviton correlation functions. However, some diagrams with three external gravitons are very involved. Their computation can be avoided by means of the Ward-TakahashiSlavnov-Taylor (WTST) identities [11-14]. The most popular technique to achieve this goal is the background field method [15-18], which incorporates the WTST identities by gauge fixing the theory in a clever way. A more standard approach is the one pursued by Salvio and Strumia in refs. [19,20]. They replace the computations of the diagrams with three external gravitons with the computations of the (much simpler) diagrams with one external graviton and two external matter fields.

In the first part of this paper, we compute the one-loop renormalization of the theory by means of a third procedure, which does not make use of the background field method and remains within the gravity sector. We quantize the theory with the help of the BatalinVilkovisky formalism [21-23] and calculate the divergences of the graviton self energy and those of the diagrams that renormalize the symmetry transformations of the fields. This approach gives a few results that are not available in the literature, such as the nonlinear contributions to the field redefinitions of the metric tensor and the Faddeev-Popov ghosts.

Then we turn to the calculation of the absorptive part of the graviton self energy, which involves novel techniques, suggested by the properties of the fakeons. For simplicity, we work in the limit of vanishing cosmological constant. A number of tricks allow us to relate the absorptive part to the renormalization of the low-energy theory, obtained by expanding the action around the Hilbert term and treating the higher-derivative terms perturbatively. The calculation obeys the power counting of the ordinary, nonrenormalizable Einstein theory, but its outcome is convergent and consistent with unitarity, by a peculiar mechanism due to the fakeons. The final formula of the absorptive part is piecewise local, equal to a contribution due to the so-called central charge $c$ of the matter fields coupled to gravity plus a correction due to the nonminimal couplings of the scalar fields plus terms that vanish on the solutions of the field equations. The results show that the quantum gravity theory of ref. [1] gives physical predictions that differ from those of any other quantization of the same classical action [15-20,24].

We use the dimensional regularization. The paper is organized as follows. In section 2 we quantize the theory to the extent that is strictly necessary for the calculation of the one-loop renormalization. In section 3 we work out the beta functions at one loop and the renormalizations of the fields. In section 4 we complete the quantization of the theory by detailing the graviton/fakeon prescription for the propagators. Then we calculate the absorptive part of the graviton self energy by relating it to the renormalization of the theory expanded around the Hilbert term. Section 5 contains the conclusions and an outlook about generalizations of the calculations performed here.

\section{Quantum gravity in the Batalin-Vilkovisky formalism}

The strictly renormalizable theory of quantum gravity proposed in ref. [1] has action

$$
S_{\mathrm{HD}}=-\frac{\mu^{-\varepsilon}}{2 \kappa^{2}} \int \sqrt{-g}\left[2 \Lambda_{C}+\zeta R+\alpha\left(R_{\mu \nu} R^{\mu \nu}-\frac{1}{3} R^{2}\right)-\frac{\xi}{6} R^{2}\right],
$$


where $\alpha, \xi, \zeta, \Lambda_{C}$ and $\kappa$ are real constants, with $\alpha>0, \xi>0$ and $\zeta>0$, while $\mu$ is the dynamical scale and $\varepsilon=4-D, D$ being the continued spacetime dimension introduced by the dimensional regularization. The action (2.1) is quantized by means of a novel graviton/fakeon prescription, which is formulated in detail in section 4 . We skip this part for the time being, because we want to concentrate on the one-loop renormalization, which coincides with the one of the Euclidean version of the theory $[1,2]$.

To apply the procedure described in the introduction and handle the WTST identities in a compact form, we use the Batalin-Vilkovisky formalism [21-23], which is a formal refinement of the Zinn-Justin approach [25]. We collect the fields into the row

$$
\Phi^{\alpha}=\left\{g_{\mu \nu}, C^{\rho}, \bar{C}^{\sigma}, B^{\tau}\right\},
$$

where $C^{\rho}$ and $\bar{C}^{\sigma}$ are the Faddeev-Popov ghosts and antighosts of diffeomorphisms, respectively, while $B^{\tau}$ are the Lagrange multipliers for the gauge fixing (also known as NakanishiLautrup fields [26, 27]). We also introduce a row of external sources

$$
K_{\alpha}=\left\{K^{\mu \nu}, K_{\rho}^{C}, K_{\sigma}^{\bar{C}}, K_{\tau}^{B}\right\},
$$

conjugate to the fields, and define the antiparentheses of two functionals $X$ and $Y$ of $\Phi$ and $K$ as

$$
(X, Y) \equiv \int\left(\frac{\delta_{r} X}{\delta \Phi^{\alpha}} \frac{\delta_{l} Y}{\delta K_{\alpha}}-\frac{\delta_{r} X}{\delta K_{\alpha}} \frac{\delta_{l} Y}{\delta \Phi^{\alpha}}\right)
$$

where the integral is over the spacetime points associated with repeated indices and the subscripts $l, r$ in $\delta_{l}, \delta_{r}$ denote the left and right functional derivatives, respectively.

The next step is to extend the action $S_{\mathrm{HD}}$ into

$$
S(\Phi, K)=S_{\mathrm{HD}}+\left(S_{K}, \Psi\right)+S_{K},
$$

where $\Psi(\Phi)$ is a functional of the fields, called gauge fermion, which is used to fix the gauge, while

$S_{K}=-\int \mathcal{R}^{\alpha}(\Phi) K_{\alpha}=\int\left(g_{\mu \rho} \partial_{\nu} C^{\rho}+g_{\nu \rho} \partial_{\mu} C^{\rho}+C^{\rho} \partial_{\rho} g_{\mu \nu}\right) K^{\mu \nu}+\int C^{\sigma}\left(\partial_{\sigma} C^{\rho}\right) K_{\rho}^{C}-\int B^{\sigma} K_{\sigma}^{\bar{C}}$ collects the infinitesimal symmetry transformations $\mathcal{R}^{\alpha}(\Phi)$ of the fields, coupled to the sources $K_{\alpha}$. In particular, the functions

$$
-\frac{\delta_{r} S}{\delta K^{\mu \nu}}=\mathcal{R}_{\mu \nu}(g, C) \equiv-g_{\mu \rho} \partial_{\nu} C^{\rho}-g_{\nu \rho} \partial_{\mu} C^{\rho}-C^{\rho} \partial_{\rho} g_{\mu \nu}
$$

are inherited from the infinitesimal transformations $\delta_{\Sigma} g_{\mu \nu}=\mathcal{R}_{\mu \nu}(g, \Sigma)$ of the metric tensor $g_{\mu \nu}$ under diffeomorphisms, where $\Sigma^{\rho}$ are functions of the spacetime point.

The action (2.2) satisfies the master equation (also known as Zinn-Justin equation)

$$
(S, S)=0,
$$

which collects the gauge invariance of $S_{\mathrm{HD}}$ and the closure of the symmetry transformations. The generating functional $Z$ of the correlation functions and the generating functional $W$ of the connected correlation functions are defined by the formulas

$$
Z(J, K)=\int[\mathrm{d} \Phi] \exp \left(i S(\Phi, K)+i \int \Phi^{\alpha} J_{\alpha}\right)=\exp i W(J, K) .
$$


The "quantum effective action", i.e. the generating functional $\Gamma(\Phi, K)=W(J, K)-\int \Phi^{\alpha} J_{\alpha}$ of the one-particle irreducible diagrams, is defined as the Legendre transform of $W(J, K)$ with respect to $J$, where $\Phi^{\alpha}=\delta_{r} W / \delta J_{\alpha}$. It is easy to see that (2.3) implies that $\Gamma$ satisfies an analogous master equation

$$
(\Gamma, \Gamma)=0,
$$

which collects all the WTST identities in a compact form.

By renormalizing the action (2.2) and taking advantage of the properties of the BatalinVilkovisky formalism, we can work out the beta functions without computing the renormalization of the three-graviton vertex and without introducing matter fields. It is sufficient to renormalize $S_{K}$ (which is relatively easy) and the graviton self energy (which is more demanding).

We expand the metric tensor $g_{\mu \nu}$ around the flat-space metric $\eta_{\mu \nu}=\operatorname{diag}(1,-1,-1,-1)$ by writing

$$
g_{\mu \nu}=\eta_{\mu \nu}+2 \kappa h_{\mu \nu},
$$

where $h_{\mu \nu}$ is the quantum fluctuation. We further define $h \equiv \eta^{\mu \nu} h_{\mu \nu}$. The indices of $\partial_{\mu}$, $h_{\mu \nu}$, the fields $\Phi^{\alpha}$ (except $g_{\mu \nu}$ ) and the sources $K_{\alpha}$ are raised and lowered by means of the flat-space metric. We raise and lower the indices of the covariant derivatives, the metric $g_{\mu \nu}$, the Riemann tensor and the Ricci tensor by means of $g_{\mu \nu}$.

We choose the gauge fermion

$$
\Psi=\mu^{-\varepsilon} \int \bar{C}^{\mu}(\sigma \zeta+\alpha \square)\left(\mathcal{G}_{\mu}-\frac{\kappa^{2}}{\lambda} B_{\mu}\right),
$$

where $\square=\eta^{\mu \nu} \partial_{\mu} \partial_{\nu}$ is the flat-space D'Alembertian,

$$
\mathcal{G}_{\mu}(g)=\eta^{\nu \rho} \partial_{\rho} g_{\mu \nu}-(\omega+1) \eta^{\nu \rho} \partial_{\mu} g_{\nu \rho}=2 \kappa\left[\partial_{\nu} h_{\mu}^{\nu}-(\omega+1) \partial_{\mu} h\right]
$$

is the gauge-fixing function and $\sigma, \lambda$ and $\omega$ are gauge-fixing parameters.

The gauge-fixed action reads

$$
S_{\mathrm{gf}}=S_{\mathrm{HD}}+\left(S_{K}, \Psi\right),
$$

where

$$
\left(S_{K}, \Psi\right)=\mu^{-\varepsilon} \int B^{\mu}(\sigma \zeta+\alpha \square)\left(\mathcal{G}_{\mu}-\frac{\kappa^{2}}{\lambda} B_{\mu}\right)+S_{\mathrm{gh}}
$$

and the action $S_{\text {gh }}$ of the Faddeev-Popov ghosts reads

$$
S_{\mathrm{gh}}=\mu^{-\varepsilon} \int\left[\bar{C}^{\mu} \partial^{\nu}-(\omega+1) \eta^{\mu \nu} \bar{C}^{\tau} \partial_{\tau}\right](\sigma \zeta+\alpha \square)\left[g_{\mu \rho} \partial_{\nu} C^{\rho}+g_{\nu \rho} \partial_{\mu} C^{\rho}+C^{\rho} \partial_{\rho} g_{\mu \nu}\right] .
$$

If we make the field redefinition $\bar{C}^{\prime \mu}=(\sigma \zeta+\alpha \square) \bar{C}^{\mu}$ on the antighosts, the ghost action turns into the more conventional form

$$
S_{\mathrm{gh}}=\mu^{-\varepsilon} \int\left[\bar{C}^{\prime \mu} \partial^{\nu}-(\omega+1) \eta^{\mu \nu} \bar{C}^{\prime \tau} \partial_{\tau}\right]\left[g_{\mu \rho} \partial_{\nu} C^{\rho}+g_{\nu \rho} \partial_{\mu} C^{\rho}+C^{\rho} \partial_{\rho} g_{\mu \nu}\right] .
$$

The ghost actions (2.8) and (2.9) are equivalent for our purposes of this paper. 


\section{Renormalization}

In this section we calculate the renormalization of the theory at one loop. Let $S_{\text {count }}$ denote the one-loop counterterm action. A few standard properties allow us to give $S_{\text {count }}$ an explicit form. First, it is easy to show that the master equations (2.3) and (2.4) imply the identity

$$
\left(S, S_{\text {count }}\right)=0 .
$$

Second, $S_{\text {count }}$ cannot depend on $B, K^{\bar{C}}$ and $K^{B}$, because no vertices of the action $(2.2)$ contain them, so no one-particle irreducible diagrams can be built with $B, K^{\bar{C}}$ and/or $K^{B}$ on the external legs. Third, $S$ does not depend on $K^{\mu \nu}$ and $\bar{C}^{\rho}$ separately, but only through the combination

$$
\tilde{K}^{\mu \nu}=K^{\mu \nu}+\mu^{-\varepsilon}(\sigma \zeta+\alpha \square) \int \frac{\delta \mathcal{G}_{\rho}}{\delta g_{\mu \nu}} \bar{C}^{\rho},
$$

so the same is true of $S_{\text {count }}$.

On general grounds, ${ }^{1}$ the solution of (3.1) can be written as

$$
S_{\text {count }}=\frac{\mu^{-\varepsilon}}{(4 \pi)^{2} \varepsilon} \int \sqrt{-g}\left[2 \Delta \Lambda_{C}+\Delta \zeta R+\Delta \alpha\left(R_{\mu \nu} R^{\mu \nu}-\frac{1}{3} R^{2}\right)-\frac{\Delta \xi}{6} R^{2}\right]+(S, \mathcal{F}),
$$

where $\Delta \Lambda_{C}, \Delta \zeta, \Delta \alpha$ and $\Delta \xi$ are constants and $\mathcal{F}(\Phi, K)$ is a local functional of ghost number minus one, equal to the integral of a local function of dimension three. Using (3.1), it is easy to show that $\mathcal{F}$ also depends on $K^{\mu \nu}$ and $\bar{C}^{\rho}$ via the combination $\tilde{K}^{\mu \nu}$. Then, the dimension of $\mathcal{F}$ and its ghost number imply that we can parametrize it as

$$
\mathcal{F}(\Phi, K)=\int \Delta g_{\mu \nu} \tilde{K}^{\mu \nu}+\int \Delta C^{\rho} K_{\rho}^{C},
$$

where $\Delta g_{\mu \nu}$ and $\Delta C^{\rho}$ are the renormalizations of the metric tensor and the Faddeev-Popov ghosts, respectively. They generalize the more common multiplications by wave function renormalization constants.

A straightforward calculation gives

$$
(S, \mathcal{F})=\int \frac{\delta S_{\mathrm{HD}}}{\delta g_{\mu \nu}} \Delta g_{\mu \nu}-\int \Delta \mathcal{R}_{\mu \nu} \tilde{K}^{\mu \nu}-\int \Delta \mathcal{R}^{\rho} K_{\rho}^{C}
$$

where

$$
\begin{aligned}
\Delta \mathcal{R}_{\mu \nu} & =-\left(S, \Delta g_{\mu \nu}\right)+\int \Delta g_{\alpha \beta} \frac{\delta_{l} \mathcal{R}_{\mu \nu}(g, C)}{\delta g_{\alpha \beta}}+\int \Delta C^{\tau} \frac{\delta_{l} \mathcal{R}_{\mu \nu}(g, C)}{\delta C^{\tau}}, \\
\Delta \mathcal{R}^{\rho} & =-\left(S, \Delta C^{\rho}\right)+\int \Delta C^{\tau} \frac{\delta_{l} \mathcal{R}^{\rho}(C)}{\delta C^{\tau}}
\end{aligned}
$$

\footnotetext{
${ }^{1}$ A convenient way to prove formulas (3.2) and (3.3) is by interpolating back and forth between the background field approach and the ordinary approach [28, 29].
} 

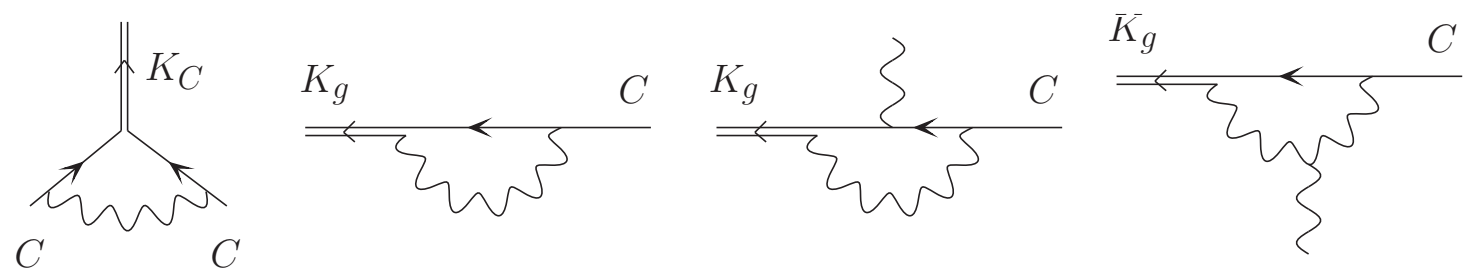

Figure 1. Diagrams that renormalize the symmetry transformations of the fields.

where $\mathcal{R}^{\rho}(C)=-\delta_{r} S / \delta K_{\rho}^{C}$. To the quadratic order in the fields, we can parametrize the field redefinitions as

$$
\begin{aligned}
\Delta C^{\rho}= & \kappa^{2} s_{1} C^{\rho}+\kappa^{3} s_{2} h_{\mu}^{\rho} C^{\mu}+\kappa^{3} s_{3} h C^{\rho} \\
\Delta g_{\mu \nu}= & \kappa^{2} t_{0} g_{\mu \nu}+\kappa^{3}\left(t_{1} h_{\mu \nu}+t_{2} \eta_{\mu \nu} h\right) \\
& +\kappa^{4}\left[t_{3} h_{\mu}^{\rho} h_{\rho \nu}+t_{4} h h_{\mu \nu}+\eta_{\mu \nu}\left(t_{5} h_{\rho \sigma} h^{\rho \sigma}+t_{6} h^{2}\right)\right],
\end{aligned}
$$

where $s_{i}$ and $t_{i}$ are constants. They can be determined by evaluating the divergent parts of the diagrams shown in figure 1, where the wiggled line denotes the field $h_{\mu \nu}$, the continuous line with the arrow denotes the Faddeev-Popov ghosts and the double lines denote either the sources $K_{g}$ coupled to the $g_{\mu \nu}$ transformations or the sources $K_{C}$ coupled to the $C$ transformations.

The calculation proceeds as follows.

1) Using (3.5), the terms proportional to $K_{\rho}^{C}$ contained in $S_{\text {count }}$ at $h_{\mu \nu}=0$ are the integral of

$$
-\Delta \mathcal{R}^{\rho} K_{\rho}^{C}=\kappa^{2}\left[\left(s_{1}+\frac{s_{2}}{2}\right) C^{\tau}\left(\partial_{\tau} C^{\rho}\right)+\frac{s_{2}}{2} C^{\tau}\left(\partial^{\rho} C_{\tau}\right)+s_{3} C^{\rho}\left(\partial_{\tau} C^{\tau}\right)\right] K_{\rho}^{C} .
$$

We can work out the values of the constants $s_{i}$ by computing the first diagram of figure 1 , which gives

$$
\Delta C^{\rho}=\frac{\kappa^{2}}{12(4 \pi)^{2} \alpha \lambda \omega^{2} \varepsilon}\left[3 C^{\rho}-8 \kappa\left(2 \omega^{2}+1\right) h_{\mu}^{\rho} C^{\mu}+\kappa\left(4 \omega^{2}-1\right) h C^{\rho}\right] .
$$

2) The terms proportional to $K^{\mu \nu}$ contained in $S_{\text {count }}$ are equal to the integral of

$$
\begin{aligned}
-\Delta \mathcal{R}_{\mu \nu} K^{\mu \nu}= & \kappa^{2}\left[\left(t_{1}-2 s_{1}\right) K^{\mu \nu} \partial_{\mu} C_{\nu}+t_{2} K \partial \cdot C\right]+2 \kappa^{3}\left(\partial_{\mu} K^{\mu \nu}\right)\left(s_{2} h_{\nu \rho} C^{\rho}+s_{3} h C_{\nu}\right) \\
& +\kappa^{3}\left[\left(t_{3}-4 s_{1}\right) K^{\mu \nu} h_{\rho \mu} \partial_{\nu} C^{\rho}-2 s_{1} K^{\mu \nu} C^{\rho} \partial_{\rho} h_{\mu \nu}+t_{3} K_{\nu}^{\mu} h_{\mu}^{\rho} \partial_{\rho} C^{\nu}+t_{4} K^{\mu \nu} h_{\mu \nu} \partial \cdot C\right. \\
& \left.+\left(t_{4}-2 t_{2}\right) K^{\mu \nu} h \partial_{\mu} C_{\nu}+2\left(t_{5}+t_{2}\right) K h_{\mu}^{\rho} \partial_{\rho} C^{\mu}+2 t_{6} K h \partial \cdot C\right]
\end{aligned}
$$

to the first order in $h_{\alpha \beta}$, where $K \equiv K_{\mu}^{\mu}$. We can work out the terms of order $\kappa^{2}$ by computing the second diagram of figure 1 , which gives

$$
\begin{aligned}
& t_{1} \pi^{2} \varepsilon=-\frac{5}{18 \alpha}-\frac{1}{3 \alpha \lambda}-\frac{1}{24 \alpha \lambda \omega^{2}}+\frac{5}{18 \alpha \omega}+\frac{1}{9 \xi}+\frac{1}{12 \xi \omega^{2}}+\frac{5}{36 \xi \omega} \\
& t_{2} \pi^{2} \varepsilon=\frac{5}{72 \alpha}-\frac{5}{48 \alpha \lambda}-\frac{1}{192 \alpha \lambda \omega^{2}}-\frac{5}{72 \alpha \omega}-\frac{1}{36 \xi}-\frac{1}{48 \xi \omega^{2}}-\frac{5}{144 \xi \omega}
\end{aligned}
$$


3) The other coefficients $t_{i}$ are obtained by computing the third and fourth diagrams of figure 1 , which give

$$
\begin{aligned}
& t_{3} \pi^{2} \varepsilon=-\frac{25}{72 \alpha}-\frac{1}{12 \alpha \lambda}-\frac{5}{72 \alpha \omega^{2}}+\frac{1}{48 \alpha \lambda \omega^{2}}+\frac{5}{12 \alpha \omega}+\frac{7}{72 \xi}+\frac{7}{144 \xi \omega^{2}}+\frac{1}{12 \xi \omega}, \\
& t_{4} \pi^{2} \varepsilon=\frac{25}{144 \alpha}+\frac{5}{144 \alpha \omega^{2}}-\frac{5}{24 \alpha \omega}-\frac{7}{144 \xi}-\frac{7}{288 \xi \omega^{2}}-\frac{1}{24 \xi \omega} \\
& t_{5} \pi^{2} \varepsilon=\frac{5}{32 \alpha}+\frac{5}{24 \alpha \lambda}+\frac{25}{288 \alpha \omega^{2}}+\frac{1}{96 \alpha \lambda \omega^{2}}+\frac{25}{144 \alpha \omega}-\frac{1}{96 \xi}-\frac{11}{576 \xi \omega^{2}}-\frac{7}{144 \xi \omega}, \\
& t_{6} \pi^{2} \varepsilon=-\frac{35}{576 \alpha}-\frac{5}{192 \alpha \omega^{2}}-\frac{5}{288 \alpha \omega}+\frac{5}{576 \xi}+\frac{1}{128 \xi \omega^{2}}+\frac{5}{288 \xi \omega} .
\end{aligned}
$$

4) A separate discussion concerns the coefficient $t_{0}$, which may be seen as the renormalization of the flat-space background metric $\eta_{\mu \nu}$ (after a redefinition of $t_{1}$ ). Observe that the contribution $\kappa^{2} t_{0} g_{\mu \nu}$ to the field redefinition $\Delta g_{\mu \nu}$ drops out of (3.7), because it is covariant. It only adds

$$
\kappa^{2} t_{0} \int \frac{\delta S_{\mathrm{HD}}}{\delta g_{\mu \nu}} g_{\mu \nu}
$$

to the first term on the right-hand side of equation (3.4). Since (3.10) is proportional to the field equations, its coefficient $t_{0}$ may be gauge dependent. However, (3.10) is also covariant, so it may mix with the renormalizations of $\Lambda_{C}, \zeta, \alpha$ and $\xi$. This means that only the combinations of such coefficients that are not affected by $t_{0}$ are truly gauge independent. It is simple to check that such combinations are $\Lambda_{C} / \zeta^{2}, \alpha$ and $\xi$. For convenience, we define

$$
t_{0} \pi^{2} \varepsilon=\frac{3}{16 \alpha \lambda}+\frac{1}{64 \alpha \lambda \omega^{2}}-\frac{3}{64 \xi \omega^{2}}-\frac{3}{16 \xi \omega}+\frac{A}{8},
$$

where $A$ is an arbitrary constant that parametrizes the surviving gauge dependence.

5) The coefficients $\Delta \Lambda_{C}, \Delta \zeta, \Delta \alpha$ and $\Delta \xi$ of $S_{\text {count }}$ can be worked out by computing the graviton self energy. We obtain

$$
\begin{aligned}
\Delta \alpha & =-\frac{133}{10}, \quad \Delta \xi=\frac{5}{6}+\frac{5 \xi}{\alpha}+\frac{5 \xi^{2}}{3 \alpha^{2}}, \quad \Delta \zeta=\zeta\left(\frac{5}{6 \xi}+\frac{5 \xi}{3 \alpha^{2}}+A\right), \\
\Delta \Lambda_{C} & =\Lambda_{C}\left(-\frac{5}{\alpha}+\frac{2}{\xi}-2 A\right)-\frac{5 \zeta^{2}}{4 \alpha^{2}}-\frac{\zeta^{2}}{4 \xi^{2}} .
\end{aligned}
$$

As promised, the combinations $\zeta \Delta \Lambda_{C}-2 \Lambda_{C} \Delta \zeta, \Delta \alpha$ and $\Delta \xi$ are independent of the arbitrary constant $A$.

The beta functions are

$$
\beta_{\alpha}=-\frac{2 \kappa^{2}}{(4 \pi)^{2}} \Delta \alpha, \quad \beta_{\xi}=-\frac{2 \kappa^{2}}{(4 \pi)^{2}} \Delta \xi, \quad \beta_{\zeta}=-\frac{2 \kappa^{2}}{(4 \pi)^{2}} \Delta \zeta, \quad \beta_{\Lambda_{C}}=-\frac{2 \kappa^{2}}{(4 \pi)^{2}} \Delta \Lambda_{C} .
$$

Now we compare our results with those of the literature. To our knowledge, the values of the coefficients $s_{i}, i=1,2,3$, encoded in formula (3.6), and those of the coefficients $t_{i}$, $i=3,4,5,6$, of formula (3.9) were not known. The most complete results for the other 
quantities are those of Salvio and Strumia, collected in ref. [20]. The notation of that paper is related to ours by the redefinitions

$$
\alpha=\frac{2}{f_{2}^{2}}, \quad \xi=\frac{2}{f_{0}^{2}}, \quad \zeta=\bar{M}_{\mathrm{Pl}}^{2}, \quad \Lambda_{C}=\Lambda, \quad \omega=\frac{c_{g}}{2}-1, \quad \lambda=-\frac{f_{2}^{2}}{\xi_{g}},
$$

together with $\sigma=0, \kappa=1$, while our $h_{\mu \nu}$ is equal to the one of [20] divided by two. Formulas (3.13) and the coefficients $t_{1}$ and $t_{2}$ of equations (3.8) agree with those of [20], apart from the following discrepancies: the term $-5 f_{2}^{4} /\left(3 f_{0}^{4}\right) \bar{M}_{\mathrm{Pl}}^{2}$ in formula (54a) of [20] should be replaced by $-5 f_{2}^{4} /\left(3 f_{0}^{2}\right) \bar{M}_{\mathrm{Pl}}^{2}$; moreover, the right-hand side of formula (55) should be multiplied by an overall minus sign and its first fraction should be multiplied by an extra factor $1 /\left(c_{g}-2\right)$. The authors of [20] have implicitly set $t_{0}=0$. This choice determines the constant $A$, which is related to the constant $X$ of [20] by the equation $A=-X-3 f_{0}^{2} / 4$.

Finally, we make a nontrivial check of $\Delta g_{\mu \nu}$ by adding $N_{s}$ scalar fields with the minimal action

$$
S_{\mathrm{s}}=\frac{1}{2} \sum_{i=1}^{N_{s}} \int \sqrt{-g} g^{\mu \nu}\left(\partial_{\mu} \varphi^{i}\right)\left(\partial_{\nu} \varphi^{i}\right) .
$$

The total action $S_{\mathrm{HD}}+S_{\mathrm{s}}$ is renormalizable. Indeed, the external scalar legs of every diagram carry derivatives, so the vertex $\sqrt{-g} \varphi^{4}$ and the nonminimal term $\sqrt{-g} R \varphi^{2}$ are not generated as counterterms, if they are absent at the classical level. No other counterterms are compatible with power counting and invariance under diffeomorphisms.

We find that the scalar self energy and the scalar-graviton vertex are renormalized by the field redefinitions $\Delta g_{\mu \nu}$ found above plus

$$
\Delta \varphi^{i}=-\frac{\kappa^{2}}{(4 \pi)^{2} \varepsilon}\left(A+\frac{3}{2 \xi}\right) \varphi^{i} .
$$

\section{Absorptive part}

In this section we calculate the absorptive part of the graviton self energy. We work in the limit $\Lambda_{C}=0$ and include $N_{s}$ scalar fields $\varphi^{i}$ coupled to gravity by the action (3.14). At the end, we add other types of matter fields. The calculation gives us the chance to show that the graviton/fakeon prescription is consistent and leads to physical predictions that are different from those obtained by quantizing the classical action (2.1) by means of standard prescriptions [15-20, 24].

For simplicity, we set the gauge-fixing parameters $\lambda$ and $\sigma$ to one, but keep $\omega$ arbitrary to check that the physical quantities we compute are gauge independent. However, due to the complications of some formulas, we report the gauge-dependent results only for $\omega=$ $-1 / 2$ (which is the de Donder gauge). The results for arbitrary $\omega$ can be downloaded from http://renormalization.com/Math/QG, together with the Mathematica programs used for the calculations of this paper.

It is convenient to integrate $B_{\mu}$ out in formula (2.7), which is equivalent to replacing it with the solution

$$
B_{\mu}=\frac{1}{2 \kappa^{2}} \mathcal{G}_{\mu}
$$


of its own field equation, or making the replacement

$$
\left(S_{K}, \Psi\right) \rightarrow \frac{\mu^{-\varepsilon}}{4 \kappa^{2}} \int \mathcal{G}^{\mu}(\zeta+\alpha \square) \mathcal{G}_{\mu}+S_{\mathrm{gh}}
$$

in formula (2.6). The free propagator of the metric fluctuation $h_{\mu \nu}$ reads

$$
\left\langle h_{\mu \nu}(p) h_{\rho \sigma}(-p)\right\rangle_{0}=\frac{i \mathcal{I}_{\mu \nu \rho \sigma}}{2 p^{2}\left(\zeta-\alpha p^{2}\right)}+\frac{i(\alpha-\xi) \varpi_{\mu \nu} \varpi_{\rho \sigma}}{6\left(p^{2}\right)^{2}\left(\zeta-\alpha p^{2}\right)\left(\zeta-\xi p^{2}\right)}
$$

at $\omega=-1 / 2$, where

$$
\mathcal{I}_{\mu \nu \rho \sigma}=\eta_{\mu \rho} \eta_{\nu \sigma}+\eta_{\mu \sigma} \eta_{\nu \rho}-\eta_{\mu \nu} \eta_{\rho \sigma}, \quad \varpi_{\mu \nu}=p^{2} \eta_{\mu \nu}+2 p_{\mu} p_{\nu} .
$$

We define the graviton/fakeon prescription by introducing two widths $\epsilon$ and $\mathcal{E}$ as follows:

(a) replace $p^{2}$ with $p^{2}+i \epsilon$ everywhere in the denominators of the propagators;

(b) turn the massive poles into fakeons by means of the replacement

$$
\frac{1}{\zeta-u\left(p^{2}+i \epsilon\right)} \rightarrow \frac{\zeta-u p^{2}}{\left(\zeta-u\left(p^{2}+i \epsilon\right)\right)^{2}+\mathcal{E}^{4}}
$$

where $u$ is equal to $\alpha$ or $\xi$.

(c) calculate the diagrams in the Euclidean framework, nonanalytically Wick rotate them as explained in refs. $[2,7,8]$, then make $\epsilon$ tend to zero first and $\mathcal{E}$ tend to zero last.

It is convenient to apply the prescription after separating the graviton poles from the fakeon poles by means of a partial fractioning. Specifically, we use formulas such as

$$
\begin{aligned}
\frac{1}{z(1-a z)} & =\frac{1}{z}+\frac{a}{1-a z}, \\
\frac{1}{z^{2}(1-a z)(1-b z)} & =\frac{1}{z^{2}}+\frac{a+b}{z}+\frac{1}{a-b}\left(\frac{a^{3}}{1-a z}-\frac{b^{3}}{1-b z}\right),
\end{aligned}
$$

etc., with $z=p^{2}, a=\alpha / \zeta$ and $b=\xi / \zeta$, to decompose the propagator (4.1) as the sum

$$
\left\langle h_{\mu \nu}(p) h_{\rho \sigma}(-p)\right\rangle_{0}=\left\langle h_{\mu \nu}(p) h_{\rho \sigma}(-p)\right\rangle_{0 \mathrm{grav}}+\left\langle h_{\mu \nu}(p) h_{\rho \sigma}(-p)\right\rangle_{0 \text { fake }}
$$

of a graviton part plus a fake part, where the graviton part collects the poles at $p^{2}=0$, while the fake part collects the poles at $p^{2}=\zeta / \alpha$ and $p^{2}=\zeta / \xi$. Once we apply the graviton/fakeon prescription as explained above, we obtain

$$
\begin{aligned}
\left\langle h_{\mu \nu}(p) h_{\rho \sigma}(-p)\right\rangle_{0 \mathrm{grav}}= & \frac{i}{2 \zeta\left(p^{2}+i \epsilon\right)}\left[\mathcal{I}_{\mu \nu \rho \sigma}+\frac{(\alpha-\xi) \varpi_{\mu \nu} \varpi_{\rho \sigma}}{3 \zeta^{2}}\left(\frac{\zeta}{p^{2}+i \epsilon}+\alpha+\xi\right)\right], \\
\left\langle h_{\mu \nu}(p) h_{\rho \sigma}(-p)\right\rangle_{0 \mathrm{fake}}= & \frac{i \alpha \mathcal{I}_{\mu \nu \rho \sigma}\left(\zeta-\alpha p^{2}\right)}{2 \zeta\left[\left(\zeta-\alpha\left(p^{2}+i \epsilon\right)\right)^{2}+\mathcal{E}^{4}\right]} \\
& +\frac{i \varpi_{\mu \nu} \varpi_{\rho \sigma}}{6 \zeta^{3}}\left(\frac{\alpha^{3}\left(\zeta-\alpha p^{2}\right)}{\left(\zeta-\alpha\left(p^{2}+i \epsilon\right)\right)^{2}+\mathcal{E}^{4}}-\frac{\xi^{3}\left(\zeta-\xi p^{2}\right)}{\left(\zeta-\xi\left(p^{2}+i \epsilon\right)\right)^{2}+\mathcal{E}^{4}}\right) .
\end{aligned}
$$


We compute the absorptive part of the graviton self energy at $\Lambda_{C}=0$. Recall that the absorptive part of an amplitude is equal to its imaginary part, so the one of a diagram is equal to minus its real part. The calculation involves three bubble diagrams with external legs $h_{\mu \nu}$. The loop can be made of scalar fields, Faddeev-Popov ghosts or $h_{\mu \nu}$ itself. The scalar contributions are not interested by the fakeons, so they coincide with those of Einstein gravity. The same conclusion applies to the contributions of the Faddeev-Popov ghosts, as is evident by working with the action (2.9). So, we focus on the bubble diagram of the metric fluctuation $h_{\mu \nu}$.

Now we prove that the fakeons do not affect the real part of this diagram, so we can drop them and replace the propagators (4.1) with $\left\langle h_{\mu \nu} h_{\rho \sigma}\right\rangle_{0 \text { grav }}$. The $h_{\mu \nu}$ bubble diagram obviously contains two propagators. Decomposing each of them as shown in formula (4.3), we obtain the sum of three terms: $(i)$ the pure graviton contributions, where each propagator is replaced by its graviton part $\left\langle h_{\mu \nu} h_{\rho \sigma}\right\rangle_{0 \text { grav }} ;(i i)$ the pure fakeon contributions, where each propagator is replaced by its fakeon part $\left\langle h_{\mu \nu} h_{\rho \sigma}\right\rangle_{0 \text { fake }} ;(i i i)$ the mixed contributions, where one propagator is replaced by its graviton part and the other propagator is replaced by the fakeon part.

The contributions of type (ii) and (iii) can be dropped, because they are purely imaginary. To see this, recall that the diagram must be calculated in the Euclidean framework and then nonanalytically Wick rotated as explained in refs. $[2,7,8]$. Moreover, we must first work at finite $\mathcal{E}$, let $\epsilon$ tend to zero while $\mathcal{E}$ is finite and nonzero, and finally let $\mathcal{E}$ also tend to zero. Varying the energy $p^{0}$ of the external momentum $p$, the poles of the propagators may pinch the integration domain. When $p^{0}$ is located below the thresholds of the pinchings, the result of the loop integral is purely imaginary. Indeed, an overall factor $i$ is brought by the residue theorem, applied to the integral on the loop energy $k^{0}$. After that, the $i \epsilon$ prescription is redundant below the thresholds, which allows us to let $\epsilon \rightarrow 0$ at the level of the integrand. Since the integrand is real in this limit, the result of the integral is purely imaginary.

In case (iii), no threshold is located on the real $p^{0}$ axis for $\mathcal{E}>0, \epsilon \rightarrow 0$, since the pinchings occur far away (at a distance roughly equal to $\mathcal{E}$ ). Then, the Wick rotation is analytic for real $p$ and the result is purely imaginary (for every $\mathcal{E}>0$ and so also when $\mathcal{E}$ tends to zero).

In case $(i i)$ the $i \epsilon$ prescription is redundant from the beginning, because only the fakeons circulate in the loop. Some thresholds of the pinchings lie on the real axis of the complex $p^{0}$ plane. Again, the result is purely imaginary below the thresholds. We can reach the regions above the thresholds by means of the average continuation $[2,7,8]$, which is the arithmetic average of the two analytic continuations that circumvent the thresholds. Clearly, the average continuation of a function that is purely imaginary in a real interval of the complex $p^{0}$ plane, is purely imaginary on the entire real $p^{0}$ axis.

In conclusion, we can concentrate on the contributions of type $(i)$, which can be evaluated by using $\left\langle h_{\mu \nu} h_{\rho \sigma}\right\rangle_{0 \text { grav }}$ as the propagator of $h_{\mu \nu}$. If we make some further steps, we can prove that the surviving contributions are uniquely determined by the divergent part of the graviton self energy, calculated in the low-energy expansion, which means expanding the action $S_{\mathrm{HD}}$ of formula (2.1) around the Hilbert term $\int \sqrt{-g} R$ and treating the param- 
eters $\alpha$ and $\xi$ perturbatively. At the same time, the result obtained with this method is exact in $\alpha$ and $\xi$.

To show these properties, we first make some observations about the expansion in question. It can be worked out by starting from the self energy diagram studied in the previous section and expanding its integrand in powers of $\alpha$ and $\xi$. Since the vertices depend on such parameters polynomially, it is sufficient to concentrate on the expansions of the propagators. When we expand the propagator (4.1), or its $\omega \neq-1 / 2$ version, we just obtain poles at $p^{2}=0$. It is sufficient to truncate the expansion of the propagator to the quadratic order in $\alpha$ and $\xi$, because higher powers simplify the poles and just multiply polynomials of the momentum. Inside the bubble diagram, these corrections give massless tadpoles (polynomials times a single massless propagator) and are set to zero by the dimensional regularization.

It is obvious [and easy to check, using formulas of type (4.2)] that the propagator, once truncated to the quadratic order in $\alpha$ and $\xi$, coincides with $\left\langle h_{\mu \nu} h_{\rho \sigma}\right\rangle_{0 \text { grav }}$, up to polynomials, which, again, are negligible for our purposes. Thus, the absorptive part of the graviton self energy can be calculated by means of the low-energy expansion. It remains to show that it is uniquely determined by the divergent part.

Since no parameters of positive dimensions in units of mass are present (the cosmological constant being set to zero), the result of the loop integral, calculated by expanding the integrand in $\alpha$ and $\xi$, must be a polynomial times $\ln \left(-p^{2}\right)$, where $p$ is the external momentum. Then it is clear that the divergent part and the absorptive part of the diagram are unambiguously related to each other. A quick way to see this is by means of the chain of relations

$$
\frac{1}{\varepsilon} \rightarrow \frac{1}{2} \ln \Lambda^{2} \rightarrow \frac{1}{2} \ln \frac{\Lambda^{2}}{-p^{2}} \rightarrow-\frac{1}{2} \ln \left(-p^{2}\right) \stackrel{\text { prescr }}{\longrightarrow}-\frac{1}{2} \ln \left(-p^{2}-i \epsilon\right) \stackrel{\text { abs }}{\longrightarrow} i \frac{\pi}{2} \theta\left(p^{2}\right)
$$

The first arrow relates the poles of the dimensional regularization to the logarithms of an ordinary cutoff $\Lambda$. The second and third arrow relate them to the logarithms of the external momentum $p$. The fourth arrow restores the Feynman prescription (which is the only prescription to be used at this point, since no fakeons have survived). The last arrow extracts the contribution to the absorptive part.

To summarize, the absorptive part of the graviton self energy can be calculated from the divergent part of the expansion in powers of $\alpha$ and $\xi$ and the result is exact in $\alpha$ and $\xi$. Thanks to this, the outcome is guaranteed to be gauge invariant (after applying field redefinitions and procedures analogous to the ones described in the previous section, adapted to the power counting of the low-energy expansion).

Note that we have slightly modified the prescription given ref. [1] to make gauge invariance manifest. Strictly speaking, the widths $\epsilon$ and $\mathcal{E}$ break gauge invariance, which must be recovered in the limit $\epsilon \rightarrow 0$ followed by $\mathcal{E} \rightarrow 0$. In general, it might be necessary to add corrections proportional to $\epsilon$ and/or $\mathcal{E}$ to implement the recovery of gauge invariance. The graviton/fakeon prescription formulated in this section is optimized to make this extra effort unnecessary. 
Let us comment on how the chain of relations (4.4) is modified in the case of the fakeons. There we have

$$
\frac{1}{\varepsilon} \rightarrow \frac{1}{2} \ln \Lambda^{2} \rightarrow \frac{1}{2} \ln \frac{\Lambda^{2}}{-p^{2}} \rightarrow-\frac{1}{2} \ln \left(-p^{2}\right) \stackrel{\text { prescr }}{\longrightarrow}-\frac{1}{4} \ln \left(-p^{2}\right)^{2} \stackrel{\text { abs }}{\longrightarrow} 0,
$$

so no absorptive part survives. The explanation of the fourth arrow can be found in ref. [1] and amounts to the fakeon prescription. In practice, the ultraviolet behavior of a two-point function is governed by two types of logarithms of the momentum. One, $\ln \left(-p^{2}-i \epsilon\right)$, is inherited from the Feynman prescription, which is associated with the physical degrees of freedom. The other one, $(1 / 2) \ln \left(p^{2}\right)^{2}$, is inherited from the fakeon prescription. From the point of view of the ultraviolet divergences, they are both equal to $-2 / \varepsilon$, which is why we had to use the tricks described above to disentangle them. Their difference gives the absorptive part, due to the identity

$$
-\ln \left(-p^{2}-i \epsilon\right)+\frac{1}{2} \ln \left(p^{2}\right)^{2}=i \pi \theta\left(p^{2}\right) .
$$

Because of the fakeons, the imaginary and real parts of a loop diagram are unrelated to each other. The divergent part obeys the renormalizable power counting of the higherderivative theory, while the absorptive part obeys the (nonrenormalizable) power counting of the low-energy expansion and is consistent with unitarity. Contributions of higher dimensions (multiplied by large powers of $\alpha$ and $\xi$ ) can appear in the absorptive part, multiplied by either side of (4.6), without affecting the divergent part. This is the basic mechanism by means of which the fakeons make renormalization and unitarity compatible with each other, in the limit of vanishing cosmological constant.

At this point, the calculation is straightforward. With the help of field redefinitions of the form

$$
\begin{aligned}
\Delta g_{\mu \nu}=\kappa^{3} \frac{i \pi}{2} \theta(-\square)[ & -2 a_{1} \square h_{\mu \nu}-a_{2} \eta_{\mu \nu} \square h-a_{3} \eta_{\mu \nu} \partial^{\rho} \partial^{\sigma} h_{\rho \sigma}-a_{4} \partial_{\mu} \partial_{\nu} h \\
& \left.-2 a_{5}\left(\partial_{\mu} \partial^{\rho} h_{\rho \nu}+\partial_{\nu} \partial^{\rho} h_{\rho \mu}\right)+a_{6} \partial_{\mu} \partial_{\nu} \partial^{\rho} \partial^{\sigma} h_{\rho \sigma}\right],
\end{aligned}
$$

where $a_{i}, i=1, \ldots 6$, are functions of $\square$, the absorptive part of the graviton self energy is encoded into the contribution

$$
\Gamma_{\mathrm{abs}}=\frac{i N_{s} \mu^{-\varepsilon}}{120(16 \pi)} \int \sqrt{-g}\left[R_{\mu \nu} \theta\left(-\square_{c}\right) R^{\mu \nu}+\frac{1}{2} R \theta\left(-\square_{c}\right) R\right]-\int \frac{\delta S_{\mathrm{HD}}}{\delta g_{\mu \nu}} \Delta g_{\mu \nu}
$$

to the functional $\Gamma$, where $\square_{c}=g^{\rho \sigma} D_{\rho} D_{\sigma}$ is the covariant D'Alembertian, $D_{\rho}$ being the covariant derivative. The coefficients of the field redefinitions (4.7) are rather lengthy, so we just report them in the simple case $\omega=-1 / 2$ :

$$
\begin{aligned}
2160 a_{1}(4 \pi)^{2} \zeta^{6}= & -\alpha\left(\alpha^{2}-\xi^{2}\right)^{2} \square^{5}-\zeta(\alpha-\xi)^{2}\left(23 \alpha^{2}+24 \alpha \xi+\xi^{2}\right) \square^{4} \\
& -2 \zeta^{2}\left(115 \alpha^{3}-99 \alpha^{2} \xi-27 \alpha \xi^{2}+11 \xi^{3}\right) \square^{3} \\
& +4 \zeta^{3}\left(89 \alpha^{2}-106 \alpha \xi+17 \xi^{2}\right) \square^{2}+72 \zeta^{4}(2 \alpha+\xi) \square-4392 \zeta^{5}, \\
3240 a_{2}(4 \pi)^{2} \zeta^{6}= & (\alpha+5 \xi)\left(\alpha^{2}-\xi^{2}\right)^{2} \square^{5}+4 \zeta(\alpha-\xi)^{2}\left(7 \alpha^{2}+6 \alpha \xi-\xi^{2}\right) \square^{4} \\
& +4 \zeta^{2}(\alpha-\xi)^{2}(55 \alpha+38 \xi) \square^{3}-48 \zeta^{3}\left(7 \alpha^{2}-53 \alpha \xi+46 \xi^{2}\right) \square^{2} \\
& +432 \zeta^{4}(3 \alpha+29 \xi) \square+9072 \zeta^{5}, \\
9\left(a_{2}+a_{3}\right)(4 \pi)^{2} \zeta^{6}= & -2 \zeta^{4}(\alpha-\xi) \square .
\end{aligned}
$$


Their $\omega$-dependent expressions can be found at http://renormalization.com/Math/QG. The other coefficients of the field redefinition remain undetermined.

Adding (massless) matter fields of all types and using the results of refs. [30-32], formula (4.8) turns into

$$
\begin{aligned}
\Gamma_{\mathrm{abs}}= & \frac{i \mu^{-\varepsilon}}{16 \pi} \int \sqrt{-g}\left[c\left(R_{\mu \nu} \theta\left(-\square_{c}\right) R^{\mu \nu}-\frac{1}{3} R \theta\left(-\square_{c}\right) R\right)+\frac{N_{s} \eta^{2}}{36} R \theta\left(-\square_{c}\right) R\right] \\
& -\int \frac{\delta S_{\mathrm{HD}}}{\delta g_{\mu \nu}} \Delta g_{\mu \nu},
\end{aligned}
$$

where $\Delta g_{\mu \nu}$ is unmodified,

$$
c=\frac{1}{120}\left(N_{s}+6 N_{f}+12 N_{v}\right)
$$

is known as "central charge" ( $N_{f}$ being the numbers of Dirac fermions plus one half the number of Weyl fermions and $N_{v}$ being the number of massless vectors) and $\eta$ is related to the coefficient of the nonminimal coupling of the scalar fields, obtained by extending (3.14) into

$$
S_{s}=\frac{1}{2} \sum_{i=1}^{N_{s}} \int \sqrt{-g}\left[g^{\mu \nu}\left(\partial_{\mu} \varphi^{i}\right)\left(\partial_{\nu} \varphi^{i}\right)+\frac{1}{6}(1+2 \eta) R \varphi^{i 2}\right] .
$$

Note that formula (4.10) is nonlocal, because it is the convergent part of an amplitude. Since the nonlocality is just due to the $\theta$ function, we can call it piecewise local. We recall that the amplitudes satisfy nonlocal WTST identities, encoded into the $\Gamma$ master equation (2.4). Although the field redefinitions and the symmetry transformations involved in such identities are nonlocal, their nonlocalities are under control, because they are generated by other kinds of amplitudes. See [33] for details on the general theory and references.

We stress again that formulas (4.8), (4.9) and (4.10) are exact in $\alpha$ and $\xi$, even if we worked them out by means of an expansion. The results (4.8) and (4.10) are gauge invariant and gauge independent, as they should, apart from the last term, which vanishes on the solutions of the $S_{\mathrm{HD}}$ field equations. In particular, we have verified that every dependence on $\omega$ can be absorbed into a suitable $\Delta g_{\mu \nu}$. On the other hand, the contributions of the matter fields cannot be absorbed into a piecewise local redefinition $\bar{\Delta} g_{\mu \nu}$ of the metric tensor, because they do not vanish on the solutions of the $S_{\mathrm{HD}}$ field equations.

It is worth to point out that when the Feynman prescription is used for all the poles of the free propagators, which is what is done in the ordinary approaches [15-20,24], the absorptive part of the graviton self energy receives nontrivial contributions from the spin-2 ghosts. This proves that the graviton/fakeon prescription leads to a different theory.

Finally, we can check that the physical degrees of freedom are indeed the graviton and the matter fields by showing that the graviton self energy satisfies the correct optical theorem. At the perturbative level, the optical theorem and the unitarity equation $S S^{\dagger}=1$ are encoded into the so-called cutting equations [34, 35]. Since the absorptive part of the graviton self energy is determined by the low-energy expansion, it satisfies the cutting equations of that expansion, which are consistent with unitarity at vanishing cosmological constant [36]. Then, the cut propagators of the complete theory, which encode the physical spectrum, coincide with those of the low-energy expansion, which are determined by the 
Hilbert term and the matter action. Thus, they receive contributions from the graviton and the matter fields, but not the fakeons.

\section{Conclusions and outlook}

In this paper we have studied the theory of quantum gravity proposed in ref. [1], by computing its renormalization at one loop and the absorptive part of the graviton self energy. The theory is the unique strictly renormalizable one of a larger class of theories, where the ghosts are eliminated by turning the poles of the free propagators that are due to the higher derivatives into fakeons. The fakeons are degrees of freedom that contribute to the correlation functions (to the extent that they make the theory renormalizable) but disappear from the physical spectrum, saving perturbative unitarity.

The renormalization coincides with the one of the Euclidean version of the theory and the results we have found are consistent with those that can be found the literature. Without making use of the background field method, we managed to save the calculation of the diagrams with three external graviton legs by computing the renormalization of the symmetry transformations and using the WTST identities. We have extended the results available in the literature by computing the first nonlinear corrections to the field renormalizations of the metric tensor and the Faddeev-Popov ghosts.

The absorptive part of the graviton self energy is a key quantity to appreciate the crucial differences between the theory of quantum gravity studied here and other quantizations of the same classical action. At zero cosmological constant, a number of tricks allow us to relate it to the renormalization of the theory expanded around the Hilbert term. The final result is the sum of a term proportional to the central charge $c$ of the matter fields coupled to gravity, plus a term due to the nonminimal coupling of the scalar fields, plus corrections that vanish on the solutions of the field equations. The correct optical theorem is satisfied, with no contributions from the fakeons.

We conclude by mentioning some interesting outlooks. With some additional effort, the calculation of the absorptive part can be extended to $\Lambda_{C} \neq 0$. However, contributions similar to those of massive tadpoles (which are divergent, but have no absorptive part) are present, so the relation (4.4) cannot be applied straightforwardly. Since the free propagator $\left\langle h_{\mu \nu} h_{\rho \sigma}\right\rangle_{0}$ has a massive scalar pole with a positive residue, another interesting possibility is to let the theory propagate an additional massive scalar field, which was implicitly turned into a fakeon in this paper.

Open Access. This article is distributed under the terms of the Creative Commons Attribution License (CC-BY 4.0), which permits any use, distribution and reproduction in any medium, provided the original author(s) and source are credited.

\section{References}

[1] D. Anselmi, On the quantum field theory of the gravitational interactions, JHEP 06 (2017) 086 [arXiv: 1704.07728] [INSPIRE].

[2] D. Anselmi, Fakeons And Lee-Wick Models, JHEP 02 (2018) 141 [arXiv:1801.00915] [INSPIRE]. 
[3] T.D. Lee and G.C. Wick, Negative Metric and the Unitarity of the S Matrix, Nucl. Phys. B 9 (1969) 209 [INSPIRE].

[4] T.D. Lee and G.C. Wick, Finite Theory of Quantum Electrodynamics, Phys. Rev. D 2 (1970) 1033 [INSPIRE].

[5] R.E. Cutkosky, P.V. Landshoff, D.I. Olive and J.C. Polkinghorne, A non-analytic S matrix, Nucl. Phys. B 12 (1969) 281 [InSPIRE].

[6] B. Grinstein, D. O'Connell and M.B. Wise, Causality as an emergent macroscopic phenomenon: The Lee-Wick O(N) model, Phys. Rev. D 79 (2009) 105019 [arXiv:0805.2156] [INSPIRE].

[7] D. Anselmi and M. Piva, A new formulation of Lee-Wick quantum field theory, JHEP 06 (2017) 066 [arXiv: 1703.04584] [INSPIRE].

[8] D. Anselmi and M. Piva, Perturbative unitarity of Lee-Wick quantum field theory, Phys. Rev. D 96 (2017) 045009 [arXiv: 1703.05563] [INSPIRE].

[9] S.B. Giddings, The boundary $S$ matrix and the AdS to CFT dictionary, Phys. Rev. Lett. 83 (1999) 2707 [hep-th/9903048] [INSPIRE].

[10] V. Balasubramanian, S.B. Giddings and A.E. Lawrence, What do CFTs tell us about Anti-de Sitter space-times?, JHEP 03 (1999) 001 [hep-th/9902052] [INSPIRE].

[11] J.C. Ward, An Identity in Quantum Electrodynamics, Phys. Rev. 78 (1950) 182 [InSPIRE].

[12] Y. Takahashi, On the generalized Ward identity, Nuovo Cim. 6 (1957) 371 [INSPIRE].

[13] A.A. Slavnov, Ward Identities in Gauge Theories, Theor. Math. Phys. 10 (1972) 99 [INSPIRE].

[14] J.C. Taylor, Ward Identities and Charge Renormalization of the Yang-Mills Field, Nucl. Phys. B 33 (1971) 436 [INSPIRE].

[15] J. Julve and M. Tonin, Quantum Gravity with Higher Derivative Terms, Nuovo Cim. B 46 (1978) 137 [INSPIRE].

[16] E.S. Fradkin and A.A. Tseytlin, Renormalizable asymptotically free quantum theory of gravity, Nucl. Phys. B 201 (1982) 469 [inSPIRE].

[17] I.G. Avramidi and A.O. Barvinsky, Asymptotic freedom in higher derivative quantum gravity, Phys. Lett. 159B (1985) 269 [INSPIRE].

[18] N. Ohta, R. Percacci and A.D. Pereira, Gauges and functional measures in quantum gravity II: Higher derivative gravity, Eur. Phys. J. C 77 (2017) 611 [arXiv:1610.07991] [InSPIRE].

[19] A. Salvio and A. Strumia, Agravity, JHEP 06 (2014) 080 [arXiv:1403.4226] [INSPIRE].

[20] A. Salvio and A. Strumia, Agravity up to infinite energy, Eur. Phys. J. C 78 (2018) 124 [arXiv: 1705. 03896] [INSPIRE].

[21] I.A. Batalin and G.A. Vilkovisky, Gauge Algebra and Quantization, Phys. Lett. 102B (1981) 27 [INSPIRE].

[22] I.A. Batalin and G.A. Vilkovisky, Quantization of Gauge Theories with Linearly Dependent Generators, Phys. Rev. D 28 (1983) 2567 [Erratum ibid. D 30 (1984) 508] [INSPIRE].

[23] S. Weinberg, The quantum theory of fields, volume 2, Cambridge University Press, Cambridge (1995). 
[24] K.S. Stelle, Renormalization of Higher Derivative Quantum Gravity, Phys. Rev. D 16 (1977) 953 [INSPIRE].

[25] J. Zinn-Justin, Renormalization of gauge theories, in Trends in Elementary Particle Physics, Lecture Notes in Physics, Vol. 37, H. Rollnik and K. Dietz eds., Springer-Verlag, Berlin, Germany, (1975).

[26] N. Nakanishi, Indefinite metric quantum field theory, Prog. Theor. Phys. Suppl. 51 (1972) 1 [INSPIRE].

[27] B. Lautrup, Canonical quantum electrodynamics in covariant gauges, Mat. Fys. Medd. Dan. Vid. Selsk. 35 (1967) 11.

[28] D. Anselmi, Background field method and the cohomology of renormalization, Phys. Rev. D 93 (2016) 065034 [arXiv: 1511.01244] [INSPIRE].

[29] D. Anselmi, Background field method, Batalin-Vilkovisky formalism and parametric completeness of renormalization, Phys. Rev. D 89 (2014) 045004 [arXiv:1311.2704] [INSPIRE].

[30] S.J. Hathrell, Trace Anomalies and $\lambda \phi^{4}$ Theory in Curved Space, Annals Phys. 139 (1982) 136 [INSPIRE].

[31] S.J. Hathrell, Trace Anomalies and QED in Curved Space, Annals Phys. 142 (1982) 34 [INSPIRE].

[32] M.D. Freeman, The Renormalization of Nonabelian Gauge Theories in Curved Space-time, Annals Phys. 153 (1984) 339 [INSPIRE].

[33] D. Anselmi, Ward identities and gauge independence in general chiral gauge theories, Phys. Rev. D 92 (2015) 025027 [arXiv:1501.06692] [INSPIRE].

[34] R.E. Cutkosky, Singularities and discontinuities of Feynman amplitudes, J. Math. Phys. 1 (1960) 429 [INSPIRE].

[35] M.J.G. Veltman, Unitarity and causality in a renormalizable field theory with unstable particles, Physica 29 (1963) 186 [InSPIRE].

[36] D. Anselmi, Aspects of perturbative unitarity, Phys. Rev. D 94 (2016) 025028 [arXiv: 1606.06348] [INSPIRE]. 